\title{
Heterogeneous weight restoration trajectories during partial hospitalization treatment for anorexia nervosa
}

\author{
Johnny Berona $\mathrm{PhD}^{1}$ | Rebekah Richmond BA ${ }^{2}$ | Renee D. Rienecke $\mathrm{PhD}^{3,4,5}$ (1)
}

${ }^{1}$ Department of Psychiatry and Behavioral Neuroscience, The University of Chicago, Chicago, Illinois

${ }^{2}$ MUSC Friedman Center for Eating Disorders, Medical University of South Carolina, Charleston, South Carolina

${ }^{3}$ Department of Pediatrics, Medical University of South Carolina, Charleston, South Carolina

${ }^{4}$ Department of Psychiatry and Behavioral Sciences, Medical University of South Carolina, Charleston, South Carolina

${ }^{5}$ Department of Psychiatry, University of Michigan, Ann Arbor, Michigan

Correspondence

Renee D. Rienecke, Medical University of South Carolina, 261 Calhoun St., Ste.

220, Charleston, SC 29401.

Email: rienecke@musc.edu.

\begin{abstract}
Early response to treatment has been shown to predict good outcome in family-based treatment, but little is known about who responds quickly.

Objective: The purpose of the current study was to examine the short-term weight gain trajectories among youth receiving partial hospitalization program services for anorexia nervosa (AN), and to identify predictors of these trajectories.

Method: Adolescent and young adults $(n=102)$ with AN or subthreshold AN completed semistructured interviews and self-report measures on admission to a family-based partial hospitalization program. Patients participated in programming 5 days a week.

Results: Three weight gain trajectories were found to indicate slow, moderate, and rapid weight gain trajectories. All rapid responders gained at least four lbs. in the first 4 weeks of treatment, compared to $86.1 \%$ of moderate responders and $51.2 \%$ of slow responders. Patients were less likely to have a moderate or rapid response trajectory if they had a mood disorder diagnosis and higher parental expressed emotion. Additionally, the presence of compensatory behavior increased the likelihood of having a rapid response.

Discussion: Despite the sometimes chronic nature of AN, most patients fell into one of the two favorable response trajectories. The identification of these trajectories underscores the importance of considering the core disordered eating behaviors (i.e., restricting, binge eating, and purging), comorbid psychopathology, and parental expressed emotion.
\end{abstract}

\section{KEYWORDS}

adolescents, anorexia nervosa, family-based treatment, partial hospitalization program, weight trajectories, young adults

\section{1 | INTRODUCTION}

Anorexia nervosa (AN) confers one of the highest mortality rates of any form of psychopathology and is often associated with enduring medical sequelae and psychological comorbidities (Arcelus et al., 2011; Franko et al., 2013; Keshaviah et al., 2014). Naturalistic studies have found that AN symptomatology onsets in adolescence and demonstrates heterogeneous trajectories over time (Attia, 2010; Wildes, Forbush, \& Markon, 2013; Lavender et al., 2011). Clinical factors such as eating disorder severity, presence of binge-eating and compensatory behaviors, weight suppression, comorbid psychopathology, and parental expressed emotion are associated with long-term course (Bodell et al., 2016; Eisler, Simic, Russell, \& Dare, 2007; Keel, Brown, Holland, \& Bodell, 2012).
Although AN can be challenging to treat, involvement of family members can facilitate rapid and sustained weight gain among adolescents and young adults (Couturier, Kimber, \& Szatmari, 2013; Le Grange et al., 2012; Stiles-Shields, Hoste, Doyle, \& Le Grange, 2012). Family-based treatment (FBT) is now considered by many to be the first-line treatment for adolescents with $\mathrm{AN}$, and recent guidelines set forth by the National Institute for Health and Care Excellence recommend that family interventions be offered to children and adolescents with eating disorders (National Institute for Health and Care Excellence, 2017). FBT consists of three phases of treatment, the first of which focuses primarily on putting parents in charge of weight restoration and a rapid return to physical health. The efficacy of FBT has led to efforts to incorporate FBT principles into higher levels of care such as partial hospitalization programs (Girz et al., 2013; Henderson 
et al., 2014; Hoste, 2015; Ornstein et al., 2012). The way in which this is done varies from program to program, but could include efforts to increase parental empowerment by requiring parental involvement in meals and/or putting parents in charge of meal decision-making. In addition, taking an agnostic stance toward the eating disorder and having weekly FBT sessions in addition to PHP programming are ways that higher levels of care have incorporated FBT principles.

Despite the efficacy of FBT, little is known about for whom FBT works or does not work, and under what circumstances. An examination of moderators and mediators of two treatments for AN - FBT and individual adolescent focused therapy (AFT) - found that patients who scored higher on measures of eating related obsessionality and eating disorder psychopathology at baseline benefitted more from FBT than from AFT. No mediators of treatment outcome were found (Le Grange et al., 2012).

Across psychopathology, there is increasing evidence that individuals who show an early response to treatment are more likely to complete treatment and to benefit from it (Almirall \& Chronis-Tuscano, 2016). Within the context of FBT, early treatment response has been defined in several studies as gaining approximately four lbs. in the first 4 weeks of treatment (Darcy et al., 2013; Le Grange, Accurso, Lock, Agras, \& Bryson, 2014; Lock et al., 2015; Madden et al., 2015). Indeed, this outpatient metric predicts remission in FBT (Doyle et al., 2010) and is prognostic of future remission from AN (Nazar et al., 2017). The importance of early response notwithstanding, past studies have been unable to identify characteristics that differentiate patients who will and will not have a rapid response to treatment (Linardon, Brennan, \& de la Piedad Garcia, 2016). Moreover, comparable metrics for response to hospital-based treatment are lacking. Jennings et al. (2017) examined weight trajectory in an inpatient setting for 500 adult women with an AN diagnosis and found four distinct groups: weight gain, weight plateau, weight fluctuate, and treatment resistance. Hartman, Wirth, and Zeeck's (2007) study of 85 adult patients with AN demonstrated that early weight gain in weeks three and four of inpatient treatment predicted good treatment outcome.

To our knowledge, only two studies have examined heterogeneity in treatment response to partial hospitalization for AN (Bégin et al., 2013; Makhzoumi et al., 2017). Bégin's study of 61 adult women examined symptom trajectories of completers, noncompleters, betterrespondents, and poorer-respondents, but did not examine variables predicting different trajectories. Makhzoumi's study of 211 women receiving inpatient and partial hospitalization treatment found slow, optimal, and fast weight gain trajectories. History of previous hospitalization, highest lifetime BMI, restricting, binge eating, and purging differentiated trajectories. However, most (82\%) participants were adults and received approximately 2 months of treatment. Given the early onset of $\mathrm{AN}$ and shortening lengths of hospital stays, identifying shorter-term trajectories of weight gain among children and adolescents could inform our understanding of how to tailor interventions to improve treatment outcomes.

A meta-analysis found amount and quality of parental involvement to be an important moderator of psychotherapy outcomes, as parents can help maintain treatment gains by supporting skills use in the home environment (Dowell \& Ogles, 2010). Thus, an important transdiagnostic clinical factor is expressed emotion, a measure of a relative's attitudes and behaviors toward an ill family member that consists of five constructs: critical comments, emotional overinvolvement, hostility, warmth, and positive remarks (Brown, Birley, \& Wing, 1972). Higher levels of expressed emotion are associated with poor response to treatment, premature treatment dropout, or posttreatment relapse for a number of psychiatric disorders (Butzlaff \& Hooley, 1998; Hooley, 2007; Le Grange, Eisler, Dare, \& Hodes, 1992; Rienecke, Accurso, Lock, \& Le Grange, 2016; Szmukler, Eisler, Russell, \& Dare, 1985; van Furth et al., 1996). In a study comparing FBT delivered to the patient with the family present versus separately, high levels of maternal criticism were associated with worse outcome when patients were seen together with family at end of treatment and five-year follow-up (Eisler et al., 2000, 2007). Similarly, in a randomized controlled trial comparing FBT and individual adolescentfocused therapy, high levels of maternal criticism were associated with premature treatment dropout, and patients with mothers scoring high on hostility gained more weight in individual therapy than in FBT (Rienecke et al., 2016). Thus, expressed emotion plays an important role in treatment outcome for patients with eating disorders, and may be particularly relevant in family-based approaches.

The primary aims of this study are twofold: (a) to examine the short-term weight gain trajectories among youth receiving partial hospitalization program services for $\mathrm{AN}$ and (b) to identify individual- and family-level predictors of these trajectories. It is hypothesized that two trajectories will emerge (i.e., rapid weight gain vs. slow weight gain) and that more rapid weight gain will be predicted by less severe eating disorder symptomatology, weight suppression, fewer hospitalizations, fewer comorbid diagnoses, and less parental expressed emotion.

\section{2 | METHOD}

\section{1 | Participants}

The participants in this study were 102 adolescents and young adults, with diagnoses of $\mathrm{AN}$ (76.5\%; $n=78$ ) or subthreshold AN (23.5\%; $n=24$ ) (defined as losing a significant amount of weight but being over the DSM-IV 85\% weight criterion). Diagnoses were made according to DSM-IV criteria by the treatment team after the initial intake appointment. Patients were on average (SD) 16.4 (2.9) years old (range 11-24) with body mass index (BMI) of 17.5 (1.6) (range 12.8-25.3) and a duration of illness of 18.3 (20.8) months. The majority of patients were female (92.2\%), Caucasian (93.1\%), and from intact families (78.4\%). A substantial proportion $(40.2 \% ; n=41)$ reported previous inpatient treatment of their eating disorder, either medically or psychiatrically. Participants completed an average of 27.8 (4.7) days of treatment. The median and modal number of treatment days was 29 with $79.4 \%$ of participants completing 29-30 days. Most patients ( $n=87$; 85.3\%) completed the average treatment length of 6 weeks. Treatment dropout was not associated with weight gain trajectory, $\chi^{2}(2, N=102)=3.37, p=.19$.

\section{2 | Treatment setting}

Patients participated in a family-based partial hospitalization program (PHP) Monday through Friday for 6 hrs per day. PHP was the entry 
level into the eating disorder program. Programming consisted of two meals, a snack, and group therapy. Elements of FBT were interwoven throughout program activities with an emphasis on phase 1 strategies to address weight restoration: parents made all food choices for their child while in the program, parents' daily attendance at breakfast with their child was required, parents attended a "debriefing" group session designed to replicate phase 1 FBT sessions, all families participated in the first two sessions of manualized FBT prior to joining the PHP, and all parents attended a weekly skills group designed to help them be more effective in supporting their child and managing eating disorder behaviors (Hoste, 2015). Skills covered included effective communication strategies and reducing expressed emotion.

\section{3 | Procedure}

Participants completed a number of semi-structured interviews and self-report measures at intake. Weight was taken by program staff daily, in light indoor clothing without shoes. Informed consent to IRBapproved study protocols was obtained from parents and patients over the age of 18 , and informed assent was obtained for patients under the age of 18.

Measures.

\subsubsection{Eating disorder examination questionnaire}

The Eating Disorder Examination Questionnaire (EDE-Q) is a 36-item self-report measure of eating disorder psychopathology (Fairburn \& Beglin, 1994). The EDE-Q has a global score and four subscales: restraint, shape concern, weight concern, and eating concern. Three participants did not have EDE-Q data available. For these individuals, eating pathology data were obtained via the Eating Disorder Examination. Weight suppression was measured as the difference between the highest and current self-reported weight. The EDE-Q has good concurrent and discriminant validity and good test-retest reliability (Black \& Wilson, 1996; Fairburn \& Beglin, 1994; Luce \& Crowther, 1999; Wilson, Nonas, \& Rosenblum, 1993).

\subsection{2 | Family questionnaire}

The Family Questionnaire is completed by parents and is a 20-item self-report measure assessing two expressed emotion (EE) components: critical comments (CC) and emotional overinvolvement (EOI). Critical comments refer to unfavorable comments about the personality or behavior of the patient. Emotional overinvolvement can refer to overly self-sacrificing behaviors or exaggerated emotional responses on the part of the parent. Possible total scores range from 20 to 80 with higher scores indicating more expressed emotion. The measure has good construct validity (Wiedemann, Rayki, Feinstein, \& Hahlweg, 2002). When data were present for both parents, the highest score was used to indicate the peak severity of expressed emotion. When data were present for neither parent $(n=5)$, data were mean imputed. Participants with and without EE data did not differ on demographic characteristics or variables of interest.

\subsubsection{MINI international neuropsychiatric interview (MINI) and Mini international neuropsychiatric interview for children and adolescents (MINI KID)}

The MINI and MINI KID (Sheehan et al., 1998; Sheehan et al., 2010) are structured diagnostic interviews used to assess current and lifetime psychopathology. Interviews were administered at intake by trained staff members, overseen by a licensed clinical psychologist. The MINI was administered to patients ages 18 and above and the MINI KID was administered to patients under the age of 18. The MINI and MINI KID have shown good reliability and concurrent validity with other structured diagnostic interviews among adult and child populations (Lecrubier et al., 1997; Sheehan et al., 1998; Sheehan et al., 2010).

\subsection{Data analysis}

Distinct trajectories of weight gain were identified within a latent growth curve, or mixed effects, modeling framework. Specifically, we implemented latent growth class analysis (LGCA) which allows for random effects in intercepts and slopes across participants, accommodates variability in intervals between observations, and handles missing data using maximum likelihood estimation with robust standard errors. Similar to cluster analysis, LGCA identifies subgroups of participants within a sample. Participants with at least two weight measurements were included. Weight gain trajectories were modeled over the course of 30 days enrolled in the partial hospitalization program.

Study aims were addressed in two steps. First, unconditional models (i.e., without covariates) were fit to model change in weight from baseline and allowed for linear, quadratic, and cubic trends in each of five trajectory classes. The Bayesian Information Criteria (BIC) were used to identify the most parsimonious set of trajectories. When comparing across classes, lower BIC values indicate improved model fit. Consistent with recommendations, valid trajectory results were considered to have classes that included at least $10 \%$ of the overall sample, or 10 participants (Jones \& Nagin, 2007).

Second, weight gain trajectory groups were compared on baseline characteristics and weight gain by the end of treatment. Multinomial regressions were then used to identify which baseline variables predicted weight gain trajectories. Single predictor multinomial models examined bivariate associations of each predictor with the resulting latent classes. These variables were then entered into multivariate multinomial regressions. All data analyses were conducted in Stata 13.

\section{3 | RESULTS}

At baseline, participants' mean global EDE-Q score was $2.9(S D=1.6)$ with weight suppression of $23.1(S D=20.1)$ lbs. Most $(n=65 ; 63.7 \%)$ participants reported at least one instance of compensatory behavior in the past 3 months. The most common form was driven exercise ( $n=58 ; 56.9 \%$ ) followed by self-induced vomiting ( $n=19 ; 18.6 \%)$ and laxative use $(n=8 ; 7.8 \%$ ). More than a third of participants $(n=35$; $34.3 \%$ ) reported at least one instance of binge eating. Objective binge-eating episodes were endorsed by more participants $(n=30$; 


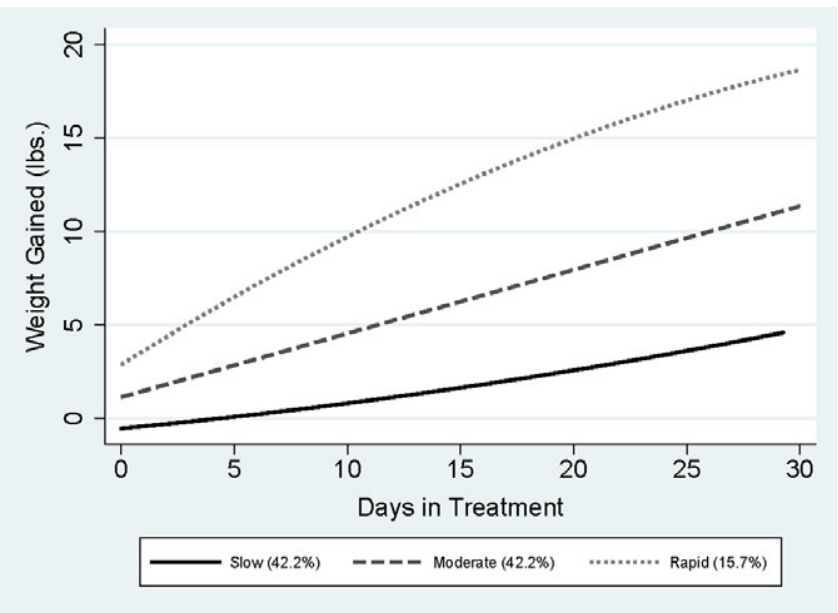

FIGURE 1 Patient treatment response trajectories while enrolled in partial hospital programming for weight restoration. Weight in pounds ( $y$-axis) is plotted against number of days in treatment ( $x$-axis). Patients with moderate ( $n=43 ; 42.2 \%$ ), steady rapid ( $n=43 ; 42.2 \%$ ), and early rapid weight gain trajectories $(n=16 ; 15.7 \%)$ are depicted [Color figure can be viewed at wileyonlinelibrary.com]

$29.4 \%$ ) than subjective binge-eating episodes ( $n=11 ; 10.8 \%$ ). On the MINI and MINI KID, more than half of participants ( $n=53$; $52.0 \%$ ) were diagnosed with a comorbid disorder. Mood disorders were slightly more prevalent $(n=39 ; 38.2 \%$ ) than anxiety disorders $(n=36$; 35.3\%). Parents' self-rated expressed emotion ranged from 29.5 to 68.0 with an average of $49.7(S D=7.6)$.

The optimal models included three distinct classes (BIC range: $-2,626.44,-2,644.09)$, providing a closer fit than a model assuming homogeneity across participants ( $\mathrm{BIC}=-3,342.42$ ). Among models with three solutions (see Figure 1), the most parsimonious solution included: (a) a quadratic growth class with a slow weight gain trajectory ( $n=43,42.2 \%$ ); (b) a linear growth class with a moderate weight gain trajectory ( $n=43,42.2 \%$ ); and (c) a quadratic growth class with a rapid weight gain trajectory $(n=16,15.7 \%)$.

The rapid weight gain group had a lower baseline BMI (mean $[S D]=16.3$ [1.4]) than the moderate (17.4 [2.1]) and slow weight gain groups $(18.1[2.5]), F(2,99)=4.07, p<.05$. The classes also differed significantly in rates of comorbid mood disorders, $\chi^{2}(2,102)=11.3$, $p<.01$. The slow weight gain trajectory class reported higher rates of comorbid mood disorders (55.81\%) compared to the moderate (30.2\%) and rapid classes (12.5\%). Groups did not differ significantly by comorbid anxiety disorder, global eating disorder severity, number of subjective and objective binge-eating episodes in the past 3 months, number of compensatory behavior episodes in the past 3 months, weight suppression, or history of prior hospitalization. All rapid responders gained at least four lbs. in the first 4 weeks of treatment compared to $86.1 \%$ of moderate responders and $51.2 \%$ of slow responders, $\chi^{2}(2,102)=20.3, p<.001$. Specifically, the rapid weight gain trajectory class gained an average of 16.7 (3.4) lbs. compared to 8.6 (3.0) lbs. gained in the moderate class and $3.1(2.2) \mathrm{lbs}$. in the slow class, $F(2,99) 144.84, p<.001$.

In bivariate regressions, associations between the above variables were examined as predictors of trajectory class membership (see Table 1). In all models, the slow weight gain trajectory served as the reference group. Global eating disorder severity, comorbid mood disorder, and expressed emotion were associated with membership in the moderate and rapid weight gain trajectory groups. Additionally, $\mathrm{BMI}$ and a comorbid anxiety disorder were associated with a rapid response. All relationships were in the expected direction. That is, markers of greater severity decreased likelihood of membership in a favorable treatment response trajectory. ${ }^{1}$

In a multivariate model including all variables, patients were less likely to have a moderate response trajectory if they had a mood disorder diagnosis (odds ratio $[\mathrm{OR}]=.39,95 \% \mathrm{Cl} . .15-1.15, p<.10$ ) and higher parental expressed emotion (OR $=.93,95 \% \mathrm{Cl}$ : .87-.99, $p<.05$ ). Additionally, the presence of compensatory behavior increased the likelihood of having a rapid response $(\mathrm{OR}=1.03,95 \% \mathrm{Cl}$ : 1.00-1.05, $p<.05)$ whereas the presence of a mood disorder diagnosis (OR $=.11,95 \% \mathrm{Cl}$ : $.02-.76, p<.05)$ reduced likelihood of rapid response.

\section{DISCUSSION}

We examined heterogeneous trajectories of weight gain in a sample of 102 adolescents and young adults enrolled in an FBT-informed

TABLE 1 Multinomial regression predicting weight gain trajectories

\begin{tabular}{|c|c|c|c|c|}
\hline \multirow[b]{3}{*}{ Variable } & \multicolumn{4}{|c|}{ Odds ratio ( $95 \%$ confidence interval) } \\
\hline & \multicolumn{2}{|c|}{ Moderate response } & \multicolumn{2}{|l|}{ Rapid response } \\
\hline & Bivariate & Multivariate & Bivariate & Multivariate \\
\hline Global eating disorder severity & $.74(.56, .98) * *$ & $.75(.52,1.09)$ & $.81(.57,1.17)$ & $.82(.47,1.42)$ \\
\hline Binge eating & $1.00(98,1.03)$ & $1.12(.97,1.04)$ & $.99(.95,1.04)$ & $.99(.95,1.06)$ \\
\hline Compensatory behavior & $.99(.98,1.01)$ & $1.00(.98,1.02)$ & $1.00(.99,1.02)$ & $1.03(1.00,1.05) * *$ \\
\hline Previous hospitalization & $1.62(.68,3.86)$ & $2.34(.83,6.54)$ & $1.12(.34,3.68)^{*}$ & $1.80(.39,8.24)$ \\
\hline Mood disorder & $.34(.14, .83)^{* *}$ & $.41(.15,1.15) *$ & $.11(.02, .56) * * *$ & $.11(.02, .76) * *$ \\
\hline Anxiety disorder & $.49(.21,1.21)$ & $.58(.20,1.71)$ & $.27(.07,1.07)^{*}$ & $.29(.05,1.74)$ \\
\hline Expressed emotion & $.96(.91,1.01)$ & $.93(.87, .99) * *$ & $.95(.88,1.03)$ & $.93(.84,1.05)$ \\
\hline
\end{tabular}

Notes. Bivariate models include only one predictor of weight gain trajectories. Multivariate models include all variables. The Slow Response trajectory is the reference group.

$* p<.10 . * * p<.05 . * * * p<.01$. 
PHP for AN. This is the first study, to our knowledge, to identify heterogeneous weight gain trajectories within the context of a PHP. Contrary to hypotheses, three distinct trajectories emerged: slow, moderate, and rapid. The slow weight gain group comprised nearly half of the sample and had the highest rate of comorbid mood disorders. The other two trajectories were significantly more likely to have gained at least four lbs. in the first 4 weeks. When attempting to identify baseline factors that were associated with trajectories, more compensatory behavior episodes, absence of a comorbid mood disorder, and lower parental expressed emotion were associated with membership in the moderate and rapid weight gain groups.

These results suggest that a PHP informed by FBT principles is generally associated with weight gain. Beyond feasibility, results are tentatively encouraging insofar as the majority of patients achieved a moderate or rapid response, which may be an important predictor of eventual remission and recovery. Given the sometimes chronic and recalcitrant nature of AN (Steinhausen, 2002), expanding the range of evidence-based interventions is crucial. Findings suggest that a PHP based on FBT principles may be an effective intervention between outpatient and inpatient treatment for achieving weight restoration.

Findings are similar to those of the broader literature indicating that a substantial proportion of those in treatment for AN have a low response to treatment (Steinhausen, 2002) and that comorbid mood disorders and higher parental expressed emotion may negatively impact treatment outcome (Eisler et al., 2007; Hughes et al., 2013; Le Grange et al., 1992; Rienecke et al., 2016; Szmukler et al., 1985; van Furth et al., 1996). A particularly notable finding was that compensatory behavior was positively associated with rapid response. This observation was present in Makhzoumi's study (2017) but does differ from a previous examination of moderators and mediators in a randomized controlled trial of FBT (Le Grange et al., 2012). In an examination of moderators and mediators, a binge-eating/purging subtype of AN was associated with poor outcomes at follow-up. Some methodological differences may explain these unanticipated findings. Patients in this study were intensively monitored in a PHP setting whereas the intervention trial was conducted in an outpatient setting. Further, in the current study, weight was intensively monitored over the course of approximately 30 days whereas the length of treatment and follow-up varied for the intervention trial. In addition, in the intervention trial, the binge/purge subtype was associated with more pervasive eating disorder and general psychopathology (e.g., higher YBC-ED and BDI scores). More broadly, previous studies have found that AN-binge/purge subtype is significantly more likely to diagnostically cross-over into BN than AN-restricting type (Keel et al., 2012). Within our study, we examined compensatory behavior episodes separately from binge-eating episodes. The presence of binge eating may be a marker for greater fluctuation in weight gain because patients may have difficulty in maintaining restricting behaviors in the

\footnotetext{
${ }^{1}$ Sensitivity analyses included two alternate multinomial regression models with a variable indicating anorexia nervosa subtype (i.e., restricting or binge-eating/ purging type). The first model included subtype in addition to number of bingeeating and compensatory behavior episodes. The second model included only the subtype variable. As subtype was not a significant predictor in any models, we report the original models. Sensitivity analyses are available from authors upon request.
}

short-term and instead rely on persistent compensatory behaviors. However, the presence of compensatory behaviors may be associated with more comorbid conditions in the long-term due to trait impulsivity or emotion dysregulation. Future studies should examine whether this particular group of youth are able to sustain treatment gains.

Findings that the absence of a comorbid mood disorder was associated with rapid weight gain is in contrast to a study finding that a diagnosis of major depression did not influence the outcome of treatment for those with AN (Calugi, El Ghoch, Conti, \& Dalle Grave, 2014). However, the study assessed inpatient as opposed to intensive outpatient treatment in a sample of adults rather than adolescents. It has been suggested that there is currently insufficient evidence to conclude that depression has a negative impact on treatment outcome for children and adolescents (Hughes, 2012). The current study suggests that absence of a mood disorder may lead to earlier weight gain in a PHP for adolescents and young adults with AN. The influence of comorbid psychopathology on the extent and course of weight restoration should be considered in future studies.

Expressed emotion has been shown to be related to treatment outcome across a number of mental disorders and medical illnesses (Wearden, Tarrier, Barrowclough, Zastowny, \& Rahill, 2000), and the current findings further support research showing that low parental expressed emotion facilitates recovery from AN. In the schizophrenia literature, relatives high on expressed emotion differ from parents low on expressed emotion in the attributions they make about a loved one's illness (Barrowclough \& Hooley, 2003). Critical relatives tend to perceive negative illness-related behaviors as under the personal control of the patient, rather than attributing these behaviors to the illness. In the case of AN, many parents initially struggle to understand that their child is not restricting their eating because he or she is being willful, stubborn, or difficult. An important focus of FBT is helping parents understand that patients restrict their food intake because they are in the grip of a powerful disorder that is causing them to be fearful of food and weight gain. Knowing this, parents can then attribute resistance to eating to the illness rather than to the individual, and there is evidence that attributional change is associated with emotional change (Brewin, 1994). Interventions specifically to reduce expressed emotion in eating disorders have been shown to improve patient outcome (Hibbs et al., 2015). Future research should assess the degree to which parents low on expressed emotion make favorable attributions about unwanted illness-related behavior in eating disorders in an effort to explain the link between low expressed emotion and good treatment outcome. Expressed emotion findings may also inform clinical decision-making. Eisler et al. found that patients with mothers who were high on critical comments did less well in conjoint family therapy than in separated family therapy (Eisler et al., 2007). It may be useful to assess familial expressed emotion at the beginning of PHP and, if FBT sessions are part of treatment, consider separated family therapy for those with high levels of parental criticism. It may also be useful to consider targeted interventions for patients of families who are high on EE. Lock et al. (2015) have shown that intensified treatment improves outcome for families identified as at risk for poor outcomes based on lack of early weight gain.

This study had several strengths that merit attention. Exploring the variability within the sample yielded several meaningful groups 
that were differentiated by the amount and speed of weight gain. Despite the often chronic nature of AN, most fell into one of two favorable weight gain trajectories. The identification of these trajectories underscores the importance of considering the core disordered eating behaviors (i.e., restricting, binge eating, and purging), comorbid psychopathology, and parental expressed emotion. Future studies could use adaptive designs that provide supplemental interventions to nonresponders such as AFT for patients or motivational interviewing for parents to facilitate changing their communication styles. In addition, weights were taken five times per week for approximately 30 days. This aspect of the study provided repeated measures in a short time period, providing a more granular view of changes in weight than can be detected in weekly outpatient treatment.

The findings of this study should be considered with its limitations in mind. Although the demographic distribution of the sample is comparable to many clinical studies, it is not a representative sample. In particular, there were few males in this sample, but the prevalence of AN has increased in recent years and affects adolescent boys and girls at the same rates (Swanson, Crow, Le Grange, Swendsen, \& Merikangas, 2011). There is currently a paucity of literature concerning the type of treatment response that would predict eventual remission from AN following an intensive treatment such as PHP. As such, metrics for recovery for this study were drawn from the outpatient literature. Future studies should conduct longer-term follow-up to ascertain the extent to which predictors of recovery from AN may differ across levels of care. As described above, the PHP itself has multiple components. As such, the primary mechanisms for facilitating weight gain may not have been the FBT elements of treatment. Further, the treatment itself was voluntary and necessarily shorterterm in duration. It is unclear how these findings would generalize to individuals who require a secure setting for long-term care.

The current study suggests that a PHP informed by FBT principles is generally associated with weight gain, as most patients fell into one of the two favorable response trajectories. The identification of these trajectories underscores the importance of considering the core disordered eating behaviors (i.e., restricting, binge eating, and purging), comorbid psychopathology, and parental expressed emotion.

\section{CONFLICT OF INTEREST}

Dr. Rienecke receives consulting fees from the Training Institute for Child and Adolescent Eating Disorders, LLC. Dr. Berona and Miss Richmond report no conflicts of interest.

\section{ORCID}

Renee D. Rienecke (D) https://orcid.org/0000-0003-0423-7403

\section{REFERENCES}

Almirall, D., \& Chronis-Tuscano, A. (2016). Adaptive interventions in child and adolescent mental health. Journal of Clinical Child \& Adolescent Psychology, 45, 383-395.

Arcelus, J., Mitchell, A. J., Wales, J., \& Nielsen, S. (2011). Mortality rates in patients with anorexia nervosa and other eating disorders: A meta-analysis of 36 studies. Archives of General Psychiatry, 68, 724-731.
Attia, E. (2010). Anorexia nervosa: Current status and future directions. Annual Review of Medicine, 61, 425-435.

Barrowclough, C., \& Hooley, J. M. (2003). Attributions and expressed emotion: A review. Clinical Psychology Review, 23, 849-880.

Bégin, C., Gagnon-Girouard, M.-P., Aimé, A., \& Ratté, C. (2013). Trajectories of eating and clinical symptoms over the course of a day hospital program for eating disorders. Eating Disorders: The Journal of Treatment and Prevention, 21, 249-264.

Black, C., \& Wilson, G. T. (1996). Assessment of eating disorders: Interview versus questionnaire. International Journal of Eating Disorders, 20, 43-50.

Bodell, L. P., Racine, S. E., \& Wildes, J. E. (2016). Examining weight suppression as a predictor of eating disorder symptom trajectories in anorexia nervosa. International Journal of Eating Disorders, 49, 753-763.

Brewin, C. R. (1994). Changes in attribution and expressed emotion among the relatives of patients with schizophrenia. Psychological Medicine, 24, 905-911.

Brown, G. W., Birley, J. L., \& Wing, J. K. (1972). Influence of family life on the course of schizophrenic disorders: A replication. British Journal of Psychiatry, 121, 241-258.

Butzlaff, R. L., \& Hooley, J. M. (1998). Expressed emotion and psychiatric relapse; a meta-analysis. Archives of General Psychiatry, 55, 547-552.

Calugi, S., El Ghoch, M., Conti, M., \& Dalle Grave, R. (2014). Depression and treatment outcome in anorexia nervosa. Psychiatry Research, 218, 195-200.

Couturier, J., Kimber, M., \& Szatmari, P. (2013). Efficacy of family-based treatment for adolescents with eating disorders: a systematic review and meta-analysis. International Journal of Eating Disorders, 46, 3-11.

Darcy, A. M., Bryson, S. W., Agras, W. S., Fitzpatrick, K. K., Le Grange, D., \& Lock, J. (2013). Do in-vivo behaviors predict early response in family-based treatment for anorexia nervosa? Behaviour Research and Therapy, 51, 762-766.

Dowell, K. A., \& Ogles, B. M. (2010). The effects of parent participation on child psychotherapy outcome: A meta-analytic review. Journal of Clinical Child \& Adolescent Psychology, 39, 151-162.

Eisler, I., Dare, C., Hodes, M., Russell, G., Dodge, E., \& Le Grange, D. (2000). Family therapy for adolescent anorexia nervosa: The results of a controlled comparison of two family interventions. Journal of Child Psychology and Psychiatry, 41, 727-736.

Eisler, I., Simic, M., Russell, G. F., \& Dare, C. (2007). A randomised controlled treatment trial of two forms of family therapy in adolescent anorexia nervosa: A five-year follow-up. Journal of Child Psychology and Psychiatry, 48, 552-560.

Fairburn, C. G., \& Beglin, S. J. (1994). Assessment of eating disorders: Interview or self-report questionnaire? International Journal of Eating Disorders, 16, 363-370.

Franko, D. L., Keshaviah, A., Eddy, K. T., Krishna, M., Davis, M. C., Keel, P. K., \& Herzog, D. B. (2013). A longitudinal investigation of mortality in anorexia nervosa and bulimia nervosa. American Journal of Psychiatry, 170, 917-925.

Girz, L., Robinson, A. L., Foroughe, M., Jasper, K., \& Boachie, A. (2013). Adapting family-based therapy to a day hospital programme for adolescents with eating disorders: Preliminary outcomes and trajectories of change. Journal of Family Therapy, 35, 102-120.

Hartmann, A., Wirth, C., \& Zeeck, A. (2007). Prediction of failure of inpatient treatment of anorexia nervosa from early weight gain. Psychotherapy Research, 17, 226-238.

Henderson, K., Buccholz, A., Obeid, N., Mossiere, A., Maras, D., Norris, M., ... Spettigue, W. (2014). A family-based eating disorder day treatment program for youth: Examining the clinical and statistical significance of short-term treatment outcomes. Eating Disorders, 22, 1-18.

Hibbs, R., Magill, N., Goddard, E., Rhind, C., Raenker, S., Macdonald, P., ... Treasure, J. (2015). Clinical effectiveness of a skills training intervention for caregivers in improving patient and caregiver health following in-patient treatment for severe anorexia nervosa: Pragmatic randomised controlled trial. British Journal of Psychiatry Open, 1, 56-66.

Hooley, J. M. (2007). Expressed emotion and relapse of psychopathology. Annual Review of Clinical Psychology, 3, 329-352.

Hoste, R. R. (2015). Incorporating family-based therapy principles into a partial hospitalization programme for adolescents with anorexia nervosa: Challenges and considerations. Journal of Family Therapy, 37, 41-60. 
Hughes, E. K. (2012). Comorbid depression and anxiety in childhood and adolescent anorexia nervosa: Prevalence and implications for outcome. Clinical Psychologist, 16, 15-24.

Hughes, E. K., Goldschmidt, A. B., Labuschagne, Z., Loeb, K. L., Sawyer, S. M., \& Le Grange, D. (2013). Eating disorders with and without comorbid depression and anxiety: Similarities and differences in a clinical sample of children and adolescents. European Eating Disorders Review, 21, 386-394.

Jennings, K. M., Gregas, M., \& Wolfe, B. (2017). Trajectories of change in body weight during inpatient treatment for anorexia nervosa. Journal of the American Psychiatric Nurses Association. doi:https://doi.org/10. 1177/1078390317726142. E-pub ahead of print.

Jones, B. L., \& Nagin, D. S. (2007). Advances in group-based trajectory modeling and an SAS procedure for estimating them. Sociological Methods \& Research, 35, 542-571.

Keel, P. K., Brown, T. A., Holland, L. A., \& Bodell, L. P. (2012). Empirical classification of eating disorders. Annual Review of Clinical Psychology, 8, 381-404.

Keshaviah, A., Edkins, K., Hastings, E. R., Krishna, M., Franko, D. L., Herzog, D. B., ... Eddy, K. T. (2014). Re-examining premature mortality in anorexia nervosa: A meta-analysis redux. Comprehensive Psychiatry, 55, 1773-1784.

Lavender, J. M., De Young, K. P., Franko, D. L., Eddy, K. T., Kass, A. E., Sears, M. S., \& Herzog, D. B. (2011). An investigation of the joint longitudinal trajectories of low body weight, binge eating, and purging in women with anorexia nervosa and bulimia nervosa. International Journal of Eating Disorders, 44, 679-686.

Lecrubier, Y., Sheehan, D. V., Weiller, E., Amorim, P., Bonora, I., Harnett Sheehan, K., ... Dunbar, G. C. (1997). The MINI international neuropsychiatric interview (MINI): A short diagnostic structured interview: Reliability and validity according to the CIDI. European Psychiatry, 12, 224-231.

Le Grange, D., Accurso, E. C., Lock, J., Agras, S., \& Bryson, S. W. (2014). Early weight gain predicts outcome in two treatments for adolescent anorexia nervosa. International Journal of Eating Disorders, 47, 124-129.

Le Grange, D., Eisler, I., Dare, C., \& Hodes, M. (1992). Family criticism and self-starvation: A study of expressed emotion. Journal of Family Therapy, 14, 177-192.

Le Grange, D., Lock, J., Agras, W. S., Moye, A., Bryson, S. W., Jo, B., \& Kraemer, H. C. (2012). Moderators and mediators of remission in family-based treatment and adolescent focused therapy for anorexia nervosa. Behaviour Research and Therapy, 50, 85-92.

Linardon, J., Brennan, L., \& de la Piedad Garcia, X. (2016). Rapid response to eating disorder treatment: A systematic review and meta-analysis. International Journal of Eating Disorders, 49, 905-919.

Lock, J., Le Grange, D., Agras, W. S., Fitzpatrick, K. K., Jo, B., Accurso, E., ... Stainer, M. (2015). Can adaptive treatment improve outcomes in family-based therapy for adolescents with anorexia nervosa? Feasibility and treatment effects of a multi-site treatment study. Behaviour Research and Therapy, 73, 90-95.

Luce, K. H., \& Crowther, J. H. (1999). The reliability of the eating disorder examination - Self-report questionnaire version (EDE-Q). International Journal of Eating Disorders, 25, 349-351.

Madden, S., Miskovic-Wheatley, J., Wallis, A., Kohn, M., Hay, P., \& Touyz, S. (2015). Early weight gain in family-based treatment predicts greater weight gain and remission at the end of treatment and remission at 12-month follow-up in adolescent anorexia nervosa. International Journal of Eating Disorders, 48, 919-922.

Makhzoumi, S. H., Coughlin, J. W., Schreyer, C. C., Redgrave, G. W., Pitts, S. C., \& Guarda, A. S. (2017). Weight gain trajectories in hospital-based treatment of anorexia nervosa. International Journal of Eating Disorders, 50, 266-274.
National Institute for Health and Care Excellence. (2017). Eating disorders: recognition and treatment. NICE guideline (NG69). London, UK: Author.

Nazar, B. P., Gregor, L. K., Albano, G., Marchica, A., Lo Coco, G. Cardi, V., \& Treasure, J. (2017). Early response to treatment in eating disorders: A systematic review and a diagnostic test accuracy meta-analysis. European Eating Disorders Review, 25, 67-79.

Ornstein, R., Lane-Loney, S., \& Hollenbeak, C. (2012). Clinical outcomes of a novel, family-centered partial hospitalization program for young patients with eating disorders. Eating and Weight Disorders, 17, e170-e177.

Rienecke, R. D., Accurso, E. C., Lock, J., \& Le Grange, D. (2016). Expressed emotion, family functioning, and treatment outcome for adolescents with anorexia nervosa. European Eating Disorders Review, 24, 43-51.

Sheehan, D. V., Lecrubier, Y., Sheehan, K. H., Amorim, P., Janavs, J., Weiller, E., ... Dunbar, G. C. (1998). The Mini-international neuropsychiatric interview (M.I.N.I.): The development and validation of a structured diagnostic psychiatric interview for DSM-IV and ICD-10. Journal of Clinical Psychiatry, 59, 21-32.

Sheehan, D. V., Sheehan, K. H., Shytle, R. D., Janavs, J., Bannon, Y., Rogers, J. E., ... Wilkinson, B. (2010). Reliability and validity of the Mini international neuropsychiatric interview for children and adolescents (MINI-KID). Journal of Clinical Psychiatry, 71, 313-326.

Steinhausen, H.-C. (2002). The outcome of anorexia nervosa in the $20^{\text {th }}$ century. American Journal of Psychiatry, 159, 1284-1293.

Stiles-Shields, C., Hoste, R. R., Doyle, P. M., \& Le Grange, D. (2012). A review of family-based treatment for adolescents with eating disorders. Reviews on Recent Clinical Trials, 7, 133-140.

Swanson, S. A., Crow, S. J., Le Grange, D., Swendsen, J., \& Merikangas, K. R. (2011). Prevalence and correlates of eating disorders in adolescents: Results from the National Comorbidity Survey Replication Adolescent Supplement. Archives of General Psychiatry, 68, 714-723.

Szmukler, G. I., Eisler, I., Russell, G. F., \& Dare, C. (1985). Anorexia nervosa, parental "expressed emotion" and dropping out of treatment. British Journal of Psychiatry, 147, 265-271.

van Furth, E. F., van Strien, D. C., Martina, L. M. L., van Son, M. J. M., Hendrickx, J. J. P., \& van Engeland, H. (1996). Expressed emotion and the prediction of outcome in adolescent eating disorders. International Journal of Eating Disorders, 20, 19-31.

Wearden, A. J., Tarrier, N., Barrowclough, C., Zastowny, T. R., \& Rahill, A. A. (2000). A review of expressed emotion research in health care. Clinical Psychology Review, 20, 633-666.

Wiedemann, G., Rayki, O., Feinstein, E., \& Hahlweg, K. (2002). The family questionnaire: Development and validation of a new self-report scale for assessing expressed emotion. Psychiatry Research, 109, 265-279.

Wildes, J. E., Forbush, K. T., \& Markon, K. E. (2013). Characteristics and stability of empirically derived anorexia nervosa subtypes: Towards the identification of homogeneous low-weight eating disorder phenotypes. Journal of Abnormal Psychology, 122, 1031-1041.

Wilson, G. T., Nonas, C. A., \& Rosenblum, G. D. (1993). Assessment of binge eating in obese patients. International Journal of Eating Disorders, 13, 25-33.

How to cite this article: Berona J, Richmond R, Rienecke RD. Heterogeneous weight restoration trajectories during partial hospitalization treatment for anorexia nervosa. Int J Eat Disord. 2018;51:914-920. https://doi.org/10.1002/eat.22922 